\title{
SUITE EXACTE DE MAYER-VIETORIS D'UNE EXTENSION TRIANGULAIRE
}

\author{
B. BENDIFFALAH
}

\begin{abstract}
In this article, we complete the results published in [2] using new proofs: we obtain a more general long exact sequence for the extension groups Ext $_{T}^{*}$ of the triangular algebra $T=\left[\begin{array}{cc}A M \\ 0 & B\end{array}\right]$. One may find such a long exact sequence in the works of Palmér and Roos [22], [23], but only for very special instances of $T$-modules. This extra effort allows us to show that the well-known Hochschild cohomological exact sequence of Happel ([16] and, more generally, [8] and [20]) is just a particular instance of that of Palmér-Roos, even if it seems we really need an assumption on $M$ (the same trick also appears for the homological version using the $\operatorname{Tor}_{*}^{T}$ functor). With this method, we obtain a sensible improvement for the isomorphism of [3], which computes the Hochschild cohomology of triangular algebras in several cases. At the end of the article, we relate the Palmér-Roos exact sequence with the classical (i.e. topological) Mayer-Vietoris exact sequence.
\end{abstract}

Un anneau commutatif $K$ est fixé et $\otimes=\otimes_{K}$.

Ici, toute algèbre $R$ est une algèbre associative unitaire sur $K$ : son algèbre opposée est notée $R^{o}$. Sauf mention explicite du contraire, un $R$-module est un module à gauche sur $R$; un module est un $K$-module. Idem pour les morphismes.

Si $A$ et $B$ sont deux algèbres, un $(A, B)$-bimodule $M$ est un $A \otimes_{K} B^{o}-$ module. S'il n'y a aucune ambiguïté nous parlerons du bimodule $M$. Un $A$ bimodule est un $(A, A)$-bimodule.

\section{Introduction}

La donnée d'une algèbre $R$ et d'un $R$-bimodule $M$, détermine une nouvelle algèbre $T$, "l'extension triviale" de $R$ par $M$ :

$$
T=R \times M, \quad(r, m)\left(r^{\prime}, m^{\prime}\right)=\left(r r^{\prime}, r m^{\prime}+m r^{\prime}\right) .
$$

Un exemple particulièrement intéressant est fourni par une algèbre "triangulaire": $R=A \times B$ est un produit direct de $K$-algèbres et $M$ est un $(A, B)$ bimodule, naturellement interprété comme un $A \times B$-bimodule. L'extension

Received October 30, 2003; in revised form March 30, 2007. 
triviale, ainsi obtenue, est notée matriciellement:

$$
T=\left[\begin{array}{cc}
A & M \\
0 & B
\end{array}\right], \quad\left[\begin{array}{cc}
a & m \\
0 & b
\end{array}\right]\left[\begin{array}{cc}
a^{\prime} & m^{\prime} \\
0 & b^{\prime}
\end{array}\right]=\left[\begin{array}{cc}
a a^{\prime} & a m^{\prime}+m b^{\prime} \\
0 & b b^{\prime}
\end{array}\right] .
$$

Le problème de la recherche d'une expression de la dimension homologique globale de $T$ (à gauche) en fonction de $R$ et de $M$ ([25], [11]) a été résolu, avec une très grande généralité, par Palmér et Roos [22], [23] (à compléter avec [19]): par exemple, leur hypothèse recouvre le cas de l'algèbre triangulaire 1.2 (problème de S. Chase, [7]).

En fait, [22] et [23] ne concernent pas la seule dimension globale de l'extension triviale 1.1. On y trouve aussi l'idée d'une suite exacte longue pour $\mathrm{Ext}_{T}^{*}$ qui, pour $T$ triangulaire, s'apparente à celle de Mayer-Vietoris, que l'on rencontre par exemple en topologie (cf. [9]). La suite exacte longue de Palmér-Roos nécessite cependant de fortes hypothèses sur l'un des deux modules: le corollaire 1 de [22] n'est validé que pour $g=0$, cf. [23, §5]. Dans cet article, nous établissons la suite exacte longue de Palmér-Roos, pour une paire de $T$-modules assez générale quand $T$ est triangulaire (Théorème 1 et 1 bis, $\S 3$ ): nous recouvrons les cas où $M$ est $B^{o}$-plat ou $A$-projectif ([2, Théorème 1.1 et $1.1 \mathrm{bis}]$ ). Ces hypothèses concernent un cadre important qui, outre les "onepoint extensions" et autres "co-extensions", recèle aussi l'algèbre $\phi !=\left[\begin{array}{cc}A & A \\ 0 & B\end{array}\right]$, introduite par Gerstenhaber et Schack ([12]), pour l'étude des déformations d'un morphisme $\phi: B \longrightarrow A$; dans [2], il est avéré aussi que le "cylindre" d'un morphisme de posets ([13, p. 4]) était aussi concerné.

Dans la suite, nous supposons $A, M$ et $B, K$-projectifs. Bon nombre d'invariants "homologiques" de l'algèbre 1.2 ont été calculés telle, par exemple, son homologie de Hochschild:

$$
H H_{*}(T) \cong H H_{*}(A) \oplus H H_{*}(B),
$$

ou encore, la $K$-théorie algébrique:

$$
K_{*}(T) \cong K_{*}(A) \times K_{*}(B) ;
$$

on notera que $M$ n'intervient nullement! (Il existe des formules identiques pour l'homologie et la cohomologie cycliques.) L'abondante littérature nous oblige à renvoyer à [18] pour toute référence sur ce sujet. La situation pour la cohomologie de Hochschild $H H^{*}(T)=H^{*}(T, T)$ est complètement différente: déjà dans [4], les auteurs obtenaient un isomorphisme

$$
H H^{*}\left[\begin{array}{ll}
A & A \\
0 & A
\end{array}\right] \cong H H^{*}(A)
$$

(à comparer avec 1.3); avec [3, 1.3.7 Corollaire], l'isomorphisme 1.5 est généralisé au cas où $M$ est $A$-projectif et $B=\left(\operatorname{End}_{A} M\right)^{o}$. Dans le cas général, 
nous devons nous contenter de la suite exacte de D. Happel ([16], généralisée par [8] et [20]; voir aussi [15], [17]). Il s'agit, là encore, d'une suite exacte longue à la Mayer-Vietoris qui existe aussi en homologie de Hochschild. Dans $\S 4$ (Théorème 3 ), nous montrons un résultat très curieux: la suite exacte de Happel est un cas particulier de celle de Palmér-Roos et, cela, même si celle de Happel ne nécessite pas d'hypothèse sur $M$ ! Cette nouvelle approche (cf. Corollaire 2) permet d'améliorer très sensiblement l'isomorphisme de [3].

Par soucis de complétude, nous établissons aussi des versions homologiques de la suite exacte longue de Palmér-Roos (foncteur Tor $_{*}^{T}$, avec hypothèses sur $M$ : cf. Théorème 2 et Théorème 2 bis, §3). Nous expliquons comment recouvrer, là aussi, la suite exacte de Happel "homologique" sans hypothèse sur $M(\$ 4$, Théorème 3 bis $)$.

L'article s'achève par l'application de la suite de Palmér-Roos à certains posets. Nous considérons la cohomologie (de Quillen ou de Hochschild) du cylindre d'un morphisme de posets ( $\$ 5$, Théorème 4); on retrouve en particulier [2, Théorème 3.1]. Aussi, nous obtenons une suite exacte longue en cohomologie pour chacun des recouvrements terminaux d'un poset $(\$ 5.17$, Théorème 5): il s'avère que la suite de Mayer-Vietoris est le cas particulier où le recouvrement est composé de deux sous-posets terminaux (Corollaire 3).

Remerciements. Plusieurs idées, développées ici, sont issues de [3] et de longues et édifiantes discussions avec Daniel Guin (Professeur à l'Université de Montpellier II, I3M): je l'en remercie très chaleureusement. Mes remerciements vont aussi au Referee pour des remarques et des commentaires très adroits qui ont radicalement changé la manière d'écrire les choses (comparer avec [2]); hors, en mathématique, le fond ne saurait être étranger à la forme.

\section{Représentations de l'Algèbre Triangulaire}

Nous renvoyons à [6], pour tout ce qui concerne les définitions et les propriétés élémentaires des bifoncteurs Tor $_{*}$ et Ext* .

La dimension globale d'une algèbre $R$ est notée gldim $(R)$; les dimensions projective et injective de tout $R$-module $X$ sont notées, respectivement, $\operatorname{pdim}_{R}(X)$ et $\operatorname{idim}_{R}(X)$. Ce sont, toutes trois des éléments de $\{-1,+\infty\} \cup \mathrm{N}$ (la dimension de 0 est -1 ). En particulier:

- $R$ est semi-simple si et seulement si $\operatorname{gldim}(R) \leq 0$.

- $R$ est héréditaire (à gauche) si et seulement si $\operatorname{gldim}(R) \leq 1$.

- X est projectif (resp. injectif) si et seulement si $\operatorname{pdim}_{R}(X) \leq 0$ (resp. $\left.\operatorname{idim}_{R}(X) \leq 0\right)$.

Autres conventions: la somme directe des bifoncteurs $\operatorname{Tor}_{n}^{R}$, avec $n \geq 1$, est notée $\operatorname{Tor}_{* \geq 1}^{R}$. De même, nous posons $\operatorname{Ext}_{R}^{* \geq 1}=\bigoplus_{n \geq 1} \operatorname{Ext}_{R}^{n}$. 
Si $T$ est une algèbre triangulaire (notations de 1.2), son algèbre opposée l'est aussi:

$$
T^{o} \cong\left[\begin{array}{cc}
B^{o} & M \\
0 & A^{o}
\end{array}\right] \text {. }
$$

Considérons l'idempotent $\pi=\left[\begin{array}{ll}1 & 0 \\ 0 & 0\end{array}\right] \in T$ : il décompose tout $T$-module (à gauche) $\Lambda$ en un $A$-module $Y=\pi \Lambda$ et un $B$-module $X=(1-\pi) \Lambda$, munis d'un morphisme de $A$-modules $f: M \otimes_{B} X \longrightarrow Y$ (l'action de $\left[\begin{array}{cc}0 & m \\ 0 & 0\end{array}\right], m \in M$ ). Ce que nous écrivons $\Lambda=[f]$ ou, s'il n'y pas d'ambiguité, $\Lambda=\left[\begin{array}{l}Y \\ X\end{array}\right]$ (matrice colonne). En particulier, nous avons une décomposition canonique en somme directe de $T$-modules projectifs:

$$
T=\left[\begin{array}{l}
A \\
0
\end{array}\right] \oplus\left[\begin{array}{c}
M \\
B
\end{array}\right] .
$$

Un $T^{o}$-module $\Lambda^{\prime}$ est décrit, lui, par un $B^{o}$-module $X^{\prime}$, un $A^{o}$-module $Y^{\prime}$ et un morphisme de $B^{o}$-modules $f^{\prime}: Y^{\prime} \otimes_{A} M \longrightarrow X^{\prime}$; notations: $\Lambda=$ $\left[f^{\prime}\right]=\left[Y^{\prime} X^{\prime}\right]$ (matrice ligne). Nous avons la décomposition en $T^{o}$-modules projectifs:

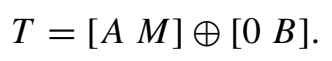

La notation matricielle est particulièrement appropriée à l'action de l'algèbre triangulaire $T$ (à gauche ou à droite). Soient à présent des $T$-modules $f$ : $M \otimes_{B} X \longrightarrow Y$ et $f^{\prime}: M \otimes_{B} X^{\prime} \longrightarrow Y^{\prime}$. La donnée d'un $T$-morphisme $\varphi:[f] \longrightarrow\left[f^{\prime}\right]$, est la donnée d'un $B$-morphisme $X \stackrel{\varphi_{X}}{\longrightarrow} X^{\prime}$ et d'un $A$ morphisme $Y \stackrel{\varphi_{Y}}{\longrightarrow} Y^{\prime}$, tels que le diagramme

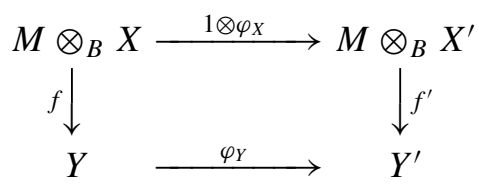

commute; notation: $\varphi=\left[\begin{array}{l}\varphi_{Y} \\ \varphi_{X}\end{array}\right]$. Nous avons les cas particuliers suivants:

$$
\operatorname{Hom}_{T}\left(\left[\begin{array}{l}
A \\
0
\end{array}\right],\left[f^{\prime}\right]\right) \cong Y^{\prime} \quad \text { et } \quad \operatorname{Hom}_{T}\left(\left[\begin{array}{c}
M \\
B
\end{array}\right],\left[f^{\prime}\right]\right) \cong X^{\prime} .
$$

Nous avons donc deux foncteurs entre les catégories abéliennes de modules à gauche $s: \operatorname{Mod}_{T} \longrightarrow \operatorname{Mod}_{A}$ et $b: \operatorname{Mod}_{T} \longrightarrow \operatorname{Mod}_{B}$, avec $s\left[\begin{array}{l}Y \\ X\end{array}\right]=Y$ et $b\left[\begin{array}{l}Y \\ X\end{array}\right]=X$. Avec 2.4, il est clair qu'une suite de $T$-modules $0 \longrightarrow\left[\begin{array}{l}Y_{1} \\ X_{1}\end{array}\right] \stackrel{\varphi}{\longrightarrow}$ $\left[\begin{array}{l}Y_{2} \\ X_{2}\end{array}\right] \stackrel{\psi}{\longrightarrow}\left[\begin{array}{l}Y_{3} \\ X_{3}\end{array}\right] \longrightarrow 0$ n'est exacte que si et seulement si celles suivantes le sont:

$$
0 \longrightarrow Y_{1} \stackrel{\varphi_{Y}}{\longrightarrow} Y_{2} \stackrel{\psi_{Y}}{\longrightarrow} Y_{3} \longrightarrow 0 \quad \text { et } \quad 0 \longrightarrow X_{1} \stackrel{\varphi_{X}}{\longrightarrow} X_{2} \stackrel{\psi_{X}}{\longrightarrow} X_{3} \longrightarrow 0 .
$$


Les foncteurs $s$ et $b$ sont donc exacts; il est également clair que le foncteur $s$ (resp. $b$ ) admet un adjoint à gauche $S: Y \longmapsto\left[\begin{array}{l}Y \\ 0\end{array}\right]$ (resp. à droite $B: X \longmapsto$ $\left[\begin{array}{l}0 \\ X\end{array}\right]$ ) aussi exact. Nous en déduisons qu'il préserve les modules injectifs (resp. projectifs) et son adjoint ceux projectifs (resp. injectifs).

2.7. Proposition 1. Nous avons des transformations naturelles de bifoncteurs:

$\operatorname{Ext}_{T}^{*}\left(\left[\begin{array}{c}Y \\ X\end{array}\right],\left[\begin{array}{c}Y^{\prime} \\ X^{\prime}\end{array}\right]\right) \stackrel{b^{*}}{\longrightarrow} \operatorname{Ext}_{A}^{*}\left(Y, Y^{\prime}\right)$ et $\operatorname{Ext}_{T}^{*}\left(\left[\begin{array}{c}Y \\ X\end{array}\right],\left[\begin{array}{c}Y^{\prime} \\ X^{\prime}\end{array}\right]\right) \stackrel{s^{*}}{\longrightarrow} \operatorname{Ext}_{B}^{*}\left(X, X^{\prime}\right)$.

2.9. Construction. Pour $n=0$, nous disposons de $s^{0}=s$ et $b^{0}=b$. Et, pour $n \geq 1$, il s'agit de leurs morphismes dérivés (cf. [6]). La construction est assez évidente: tout élément $\xi \in \operatorname{Ext}_{T}^{n}\left([f],\left[f^{\prime}\right]\right)$ est la classe d'une $T$ extension $0 \rightarrow\left[\begin{array}{l}Y^{\prime} \\ X^{\prime}\end{array}\right] \rightarrow\left[\begin{array}{l}Y_{n} \\ X_{n}\end{array}\right] \rightarrow \cdots \rightarrow\left[\begin{array}{l}Y_{1} \\ X_{1}\end{array}\right] \rightarrow\left[\begin{array}{l}Y \\ X\end{array}\right] \rightarrow 0$ qui détermine une $A$-extension $0 \rightarrow Y^{\prime} \rightarrow Y_{n} \rightarrow \cdots \rightarrow Y_{1} \rightarrow Y \rightarrow 0$ ainsi qu'une $B$ extension $0 \rightarrow X^{\prime} \rightarrow X_{n} \rightarrow \cdots \rightarrow X_{1} \rightarrow X \rightarrow 0$. De là, les éléments $s^{n}(\xi) \in \operatorname{Ext}_{A}^{n}\left(Y, Y^{\prime}\right)$ et $b^{n}(\xi) \in \operatorname{Ext}_{B}^{n}\left(X, X^{\prime}\right)$.

Par construction, les morphismes $b^{*}$ et $s^{*}$ sont compatibles au produit de Yoneda et, en particulier, nous avons un morphisme d'anneaux:

$$
\operatorname{Ext}_{T}^{*}([f],[f]) \longrightarrow \operatorname{Ext}_{A}^{*}(Y, Y) \times \operatorname{Ext}_{B}^{*}(X, X) .
$$

2.11. Proposition 2. Pour tout $T$-module projectif $\Lambda$, il existe un isomorphisme de $T$-modules $\Lambda \cong\left[\begin{array}{l}Y \\ 0\end{array}\right] \oplus\left[1_{M \otimes_{B} X}\right]$, où $Y$ est un $A$-module projectif et $X$ est un $B$-module projectif.

C'est un cas particulier de 1.4 qui affirme que le foncteur $\left(M \otimes_{B} X \stackrel{f}{\longrightarrow}\right.$ $Y) \longmapsto($ coker $f, X)$, induit un isomorphisme au niveau du groupe de Grothendieck: $K_{0}(T) \cong K_{0}(A) \times K_{0}(B)$.

Preuve. Tout $T$-module projectif est isomorphe à un facteur direct de $T^{(I)}$, pour un certain cardinal $I$. D' après 2.2 , les $T$-modules $\left[\begin{array}{c}A^{(I)} \\ 0\end{array}\right]$ et $\left[\begin{array}{c}M^{(I)} \\ B^{(I)}\end{array}\right]$ sont projectifs et tout $T$-module projectif est isomorphe à la somme d'un facteur $\left[\begin{array}{l}Y \\ 0\end{array}\right]$ de $\left[\begin{array}{c}A^{(I)} \\ 0\end{array}\right]$ et d'un facteur $\left[1_{M \otimes_{B} X}\right]$ de $\left[\begin{array}{c}M^{(I)} \\ B^{(I)}\end{array}\right]$. Nécessairement: $Y$ est un $A$-module projectif et $X$ est un $B$-module projectif.

D'après la Proposition 2, un $T$-module $M \otimes_{B} X \stackrel{f}{\longrightarrow} Y$ est projectif si et seulement s'il vérifie les trois conditions suivantes: le $B$-module $X$ est projectif, le A-morphisme $f$ est injectif et le A-module coker $(f)$ est projectif. 
La donnée d'un $T^{o}$-module $Y^{\prime} \otimes_{A} M \stackrel{f^{\prime}}{\longrightarrow} X^{\prime}$ permet de dualiser la Proposition 1. Notons que l'inclusion naturelle $A \times B \subset T$ fournit des morphismes:

$$
Y^{\prime} \otimes_{A} Y \stackrel{\tilde{b}}{\longrightarrow}\left[f^{\prime}\right] \otimes_{T}[f] \quad \text { et } \quad X^{\prime} \otimes_{B} X \stackrel{\tilde{s}}{\longrightarrow}\left[f^{\prime}\right] \otimes_{T}[f],
$$

avec les cas particuliers suivants:

$$
\begin{aligned}
{[A M] \otimes_{T}[f] \cong Y, \quad[0 B] \otimes_{T}[f] \cong X, } & \\
& {\left[f^{\prime}\right] \otimes_{T}\left[\begin{array}{c}
A \\
0
\end{array}\right] \cong Y^{\prime}, \quad\left[f^{\prime}\right] \otimes_{T}\left[\begin{array}{c}
M \\
B
\end{array}\right] \cong X^{\prime} . }
\end{aligned}
$$

La construction utilisée pour $s^{*}$ et $b^{*}$ s'adapte pour obtenir le résultat suivant:

2.14. Proposition 3. Nous avons des morphismes gradués canoniques:

$$
\operatorname{Tor}_{*}^{A}\left(Y^{\prime}, Y\right) \stackrel{\tilde{b}_{*}}{\longrightarrow} \operatorname{Tor}_{*}^{T}\left(\left[f^{\prime}\right],[f]\right) \quad \text { et } \quad \operatorname{Tor}_{*}^{B}\left(X^{\prime}, X\right) \stackrel{\tilde{s}_{*}}{\longrightarrow} \operatorname{Tor}_{*}^{T}\left(\left[f^{\prime}\right],[f]\right) .
$$

Contrairement au morphisme $(b, s)$ (et à celui $(\tilde{b}, \tilde{s}))$ le morphisme $\left(b^{*}, s^{*}\right)$ (resp. $\left.\tilde{b}_{*}+\tilde{s}_{*}\right)$ n'est plus nécessairement injectif (resp. surjectif). L'analyse des noyaux et conoyaux s'effectue grâce à des suites exactes longues de type Mayer-Vietoris.

\section{Modules d'Extensions des Algèbres Triangulaires}

Les résultats donnés ici sont en partie publiés dans [2]. Les démonstrations, inspirées des remarques du rapporteur, en sont un peu différentes.

Considérons l'algèbre triangulaire $T$ décrite dans 1.2. Étant donnés un $A$ module $Y$ et un $B$-module $X$, nous avons un isomorphisme gradué

$$
\operatorname{Ext}_{A}^{*}\left(M \otimes_{B} X, Y\right) \cong \operatorname{Ext}_{B}^{*}\left(X, \operatorname{Hom}_{A}(M, Y)\right)
$$

si $\operatorname{Tor}_{* \geq 1}^{B}(M, X)=0$ et $\operatorname{Ext}_{A}^{* \geq 1}(M, Y)=0$ (cf. [6], (4) p. 346, avec $\left.\Gamma=K\right)$. De même, pour un $A^{o}$-module $X^{\prime}$, nous avons:

$$
\operatorname{Tor}_{*}^{B}\left(X^{\prime} \otimes_{A} M, X\right) \cong \operatorname{Tor}_{*}^{A}\left(X^{\prime}, M \otimes_{B} X\right),
$$

si $\operatorname{Tor}_{* \geq 1}^{A}\left(X^{\prime}, M\right)=0$ et $\operatorname{Tor}_{* \geq 1}^{B}(M, X)=0$ (cf. [6], (4a) p.347, avec $\left.\Gamma=K\right)$.

Pour énoncer la suite exacte longue de Mayer-Vietoris "cohomologique" (Théorème 1 et Théorème $1 \mathrm{bis}$ ), nous considérons des $T$-modules décrits par des $A$-morphismes:

$$
f: M \otimes_{B} X \longrightarrow Y \quad \text { et } \quad f^{\prime}: M \otimes_{B} X^{\prime} \longrightarrow Y^{\prime} .
$$


3.4. LeMme 1. Nous avons des isomorphismes:

$$
\begin{aligned}
& b^{*}: \operatorname{Ext}_{T}^{*}\left(\left[\begin{array}{l}
Y \\
0
\end{array}\right],\left[f^{\prime}\right]\right) \cong \operatorname{Ext}_{A}^{*}\left(Y, Y^{\prime}\right) \quad \text { et } \\
& s^{*}: \operatorname{Ext}_{T}^{*}\left([f],\left[\begin{array}{c}
0 \\
X^{\prime}
\end{array}\right]\right) \cong \operatorname{Ext}_{B}^{*}\left(X, X^{\prime}\right) .
\end{aligned}
$$

3.6. Preuve. La présente est due au referee. Nous avons un foncteur exact $S: Y \longmapsto\left[\begin{array}{l}Y \\ 0\end{array}\right]$ et un isomorphisme $b^{0}: F\left(\left[\begin{array}{l}Y \\ 0\end{array}\right]\right) \cong \operatorname{Hom}_{A}\left(Y, Y^{\prime}\right)$ si $F$ est le foncteur $F\left(\left[\begin{array}{l}Y \\ X\end{array}\right]\right)=\operatorname{Hom}_{T}\left(\left[\begin{array}{c}Y \\ X\end{array}\right],\left[\begin{array}{c}Y^{\prime} \\ X^{\prime}\end{array}\right]\right)$. Puisque $S$ préserve les projectifs (2.6), nous avons $\mathbf{R}^{*}(F)(S Y) \cong \mathbf{R}^{*}(F S)(Y)$ (dérivées à droite), ce qui constitue la partie gauche de 3.5 . Pour l'isomorphisme de droite on considère le foncteur $G\left(\left[\begin{array}{c}Y^{\prime} \\ X^{\prime}\end{array}\right]\right)=\operatorname{Hom}_{T}\left(\left[\begin{array}{c}Y \\ X\end{array}\right],\left[\begin{array}{c}Y^{\prime} \\ X^{\prime}\end{array}\right]\right)$. L'exactitude du foncteur $B: X^{\prime} \longmapsto\left[\begin{array}{c}0 \\ X^{\prime}\end{array}\right]$ et le fait qu'il préserve les injectifs, permet d'étendre l'isomorphisme $s^{0}$ : $G\left(\left[\begin{array}{c}0 \\ X^{\prime}\end{array}\right]\right) \cong \operatorname{Hom}_{B}\left(X, X^{\prime}\right)$ aux dérivées à droite: $\mathbf{R}^{*}(G)\left(B X^{\prime}\right) \cong \mathbf{R}^{*}(G B)\left(X^{\prime}\right)$, soit la partie droite de 3.5 .

Avec le Lemme 1, il est facile de retrouver l'encadrement de [10]:

$\max \left\{\operatorname{gldim} A, \operatorname{gldim} B, 1+\operatorname{pdim}_{A} M\right\}$

$$
\leq \operatorname{gldim} T \leq \max \left\{\operatorname{gldim} A, \operatorname{gldim} B+1+\operatorname{pdim}_{A} M\right\}
$$

En particulier: si $B$ est semi-simple (cas des “one-point extensions") et $A \neq 0$, nous obtenons la formule de S. Chase ([7]):

$$
\operatorname{gldim}(T)=\max \left\{\operatorname{gldim}(A), 1+\operatorname{pdim}_{A}(M)\right\} .
$$

L'encadrement de [10] n'est optimal que dans le cas où $B$ est semi-simple. Dans [22] (Corollaire 4'), on trouve un meilleur encadrement qui devient, si $A$ est semi-simple ${ }^{\dagger}$,

$$
\max \left\{\operatorname{gldim}(B), 1+\operatorname{fdim}_{B^{o}}(M)\right\} \leq \operatorname{gldim}(T) \leq \operatorname{gldim}(B)+1 .
$$

3.9. LeMme 2. Si $\operatorname{Tor}_{* \geq 1}^{B}(M, X)=0$ nous avons un isomorphisme:

$$
s^{*}: \operatorname{Ext}_{T}^{*}\left(\left[1_{M \otimes_{B} X}\right],\left[f^{\prime}\right]\right) \cong \operatorname{Ext}_{B}^{*}\left(X, X^{\prime}\right) .
$$

En particulier: si $\operatorname{Tor}_{* \geq 1}^{B}(M, X)=0$ alors $\operatorname{Ext}_{T}^{*}\left(\left[1_{M \otimes_{B} X}\right],\left[\begin{array}{c}Y^{\prime} \\ 0\end{array}\right]\right)=0$.

Preuve. La démonstration s'apparente à celle du Lemme 1: là aussi, il est possible d'impliquer une suite spectrale de Grothendieck (composition de foncteurs) qui dégénère. La preuve suivante utilise 3.1. Nous avons un isomorphisme de $T$-modules $\left[1_{M \otimes_{B} X}\right] \cong\left[\begin{array}{c}M \\ B\end{array}\right] \otimes_{B} X$ et, $\left[\begin{array}{c}M \\ B\end{array}\right]$ étant $T$-projectif

\footnotetext{
(†) fdim dénote la dimension plate
} 
et $\operatorname{Tor}_{* \geq 1}^{B}(M, X)=0$, nous avons avec 3.1 un isomorphisme: $\operatorname{Ext}_{T}^{*}\left(\left[1_{M \otimes_{B} X}\right]\right.$, $\left.\left[f^{\prime}\right]\right) \cong \operatorname{Ext}_{B}^{*}\left(X, \operatorname{Hom}_{T}\left(\left[\begin{array}{c}M \\ B\end{array}\right],\left[f^{\prime}\right]\right)\right)$. Avec 2.5, on en déduit 3.10.

Nous arrivons à la première suite exacte longue de Mayer-Vietoris de l'algèbre $T$.

3.11. ThÉORÈME 1. Si $\operatorname{Tor}_{* \geq 1}^{B}(M, X)=0$, nous avons une suite exacte longue:

$$
\begin{aligned}
\cdots \longrightarrow \operatorname{Ext}_{T}^{*}\left([f],\left[f^{\prime}\right]\right) \stackrel{\left(b^{*}, s^{*}\right)}{\longrightarrow} \operatorname{Ext}_{A}^{*}\left(Y, Y^{\prime}\right) \times \operatorname{Ext}_{B}^{*}\left(X, X^{\prime}\right) & \\
& \longrightarrow \operatorname{Ext}_{A}^{*}\left(M \otimes_{B} X, Y^{\prime}\right) \longrightarrow \cdots
\end{aligned}
$$

où le morphisme $\operatorname{Ext}_{A}^{*}\left(Y, Y^{\prime}\right) \longrightarrow \operatorname{Ext}_{A}^{*}\left(M \otimes_{B} X, Y^{\prime}\right)$ est induit par $f$.

En particulier (comparer avec l'encadrement de [10]): si $M$ est $B^{\circ}$-plat nous obtenons le Théorème 1.1 de [2] ainsi que la formule suivante (cas particulier de [22], [23]):

$$
\operatorname{gldim}(T)=\max \left\{\operatorname{gldim}(B), \operatorname{gldim}(A), 1+\sup _{X} \operatorname{pdim}_{A}\left(M \otimes_{B} X\right)\right\} .
$$

PREUVE. Le diagramme commutatif et à lignes exactes suivant

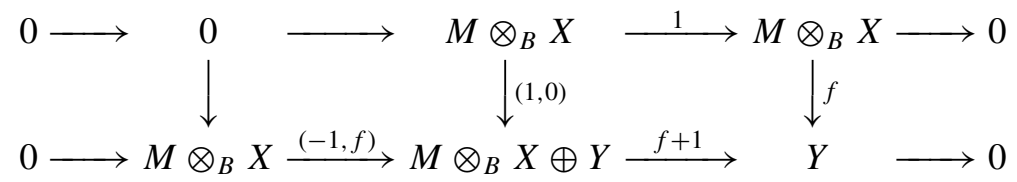

définit une suite exacte de $T$-modules: $0 \longrightarrow\left[\begin{array}{c}0 \\ M \otimes_{B} X\end{array}\right] \longrightarrow\left[1_{M \otimes_{B} X}\right] \oplus\left[\begin{array}{l}Y \\ 0\end{array}\right] \longrightarrow$ $[f] \longrightarrow 0$. Avec les Lemmes 1 et 2 , la suite exacte longue de Ext $_{T}^{*}$ prend la forme 3.12 .

3.15. Lemme 2 (bis). Le morphisme canonique $\varepsilon_{Y^{\prime}}: M \otimes_{B} \operatorname{Hom}_{A}\left(M, Y^{\prime}\right)$ $\longrightarrow Y^{\prime}$ définit naturellement un $T$-module et, si $\operatorname{Ext}_{A}^{* \geq 1}\left(M, Y^{\prime}\right)=0$, alors $b^{*}$ est un isomorphisme:

$$
\operatorname{Ext}_{T}^{*}\left([f],\left[\varepsilon_{Y^{\prime}}\right]\right) \cong \operatorname{Ext}_{A}^{*}\left(Y, Y^{\prime}\right) .
$$

Preuve. Par définition, nous avons un isomorphisme de $T$-modules, $\left[\varepsilon_{Y^{\prime}}\right]$ $\cong \operatorname{Hom}_{A}\left([A M], Y^{\prime}\right)$, où le $T^{o}$-module $[A M]$ est plat. Notre hypothèse permet d'utiliser 3.1:

$$
\operatorname{Ext}_{T}^{*}\left([f],\left[\varepsilon_{Y^{\prime}}\right]\right) \cong \operatorname{Ext}_{A}^{*}\left([A M] \otimes_{T}[f], Y^{\prime}\right),
$$

c'est-à-dire l'isomorphisme 3.16 d'après 2.13 . 
3.17. ThÉORÈme 1 (bis). Si $\operatorname{Ext}_{A}^{* \geq 1}\left(M, Y^{\prime}\right)=0$, nous avons une suite exacte longue

$$
\begin{aligned}
\cdots \longrightarrow \operatorname{Ext}_{T}^{*}\left([f],\left[f^{\prime}\right]\right) \stackrel{b^{*}}{\longrightarrow} & \operatorname{Ext}_{A}^{*}\left(Y, Y^{\prime}\right) \\
& \operatorname{Ext}_{B}^{*}\left(X, X^{\prime}\right) \\
& \longrightarrow \operatorname{Ext}_{B}^{*}\left(X, \operatorname{Hom}_{A}\left(M, Y^{\prime}\right)\right) \longrightarrow \cdots
\end{aligned}
$$

où le morphisme $\operatorname{Ext}_{B}^{*}\left(X, X^{\prime}\right) \longrightarrow \operatorname{Ext}_{B}^{*}\left(X, \operatorname{Hom}_{A}\left(M, Y^{\prime}\right)\right)$ est induit par le $B$-morphisme $\tilde{f}^{\prime}: X^{\prime} \longrightarrow \operatorname{Hom}_{A}\left(M, Y^{\prime}\right)$, adjoint de $f^{\prime}$. (La superposition signifie un produit direct.)

Si $M$ est $A$-projectif, nous obtenons le Théorème 1.1 bis de [2] et (cf. [22], [23]):

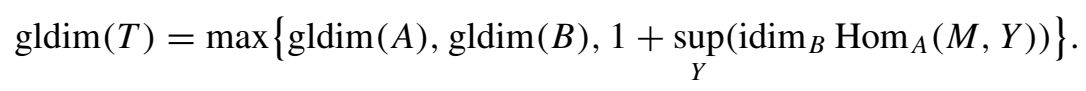

Si $A$ est semi-simple (cas des "one-point co-extensions"), nous obtenons la formule suivante:

$$
\operatorname{gldim}(T)=\max \left\{\operatorname{gldim}(B), 1+\operatorname{idim}_{B} \operatorname{Hom}_{A}(M, A)\right\},
$$

à comparer avec celle de Chase (3.7) et l'encadrement 3.8.

Preuve. La démonstration est duale de celle du Théorème 1. La suite exacte longue 3.18 provient, là aussi, d'un diagramme commutatif à lignes exactes:

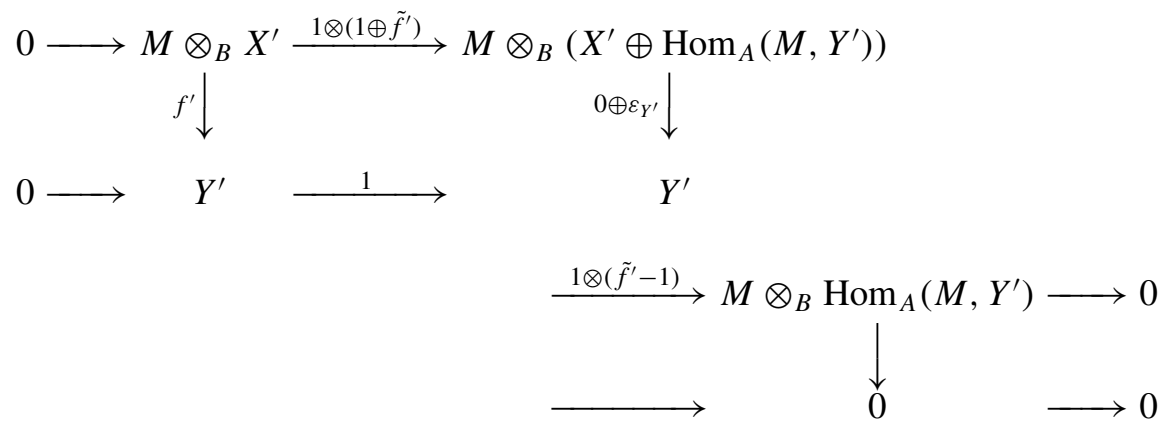

Il détermine une suite exacte courte de $T$-modules:

$$
0 \longrightarrow\left[f^{\prime}\right] \longrightarrow\left[\begin{array}{c}
0 \\
X^{\prime}
\end{array}\right] \oplus\left[\varepsilon_{Y^{\prime}}\right] \longrightarrow\left[\begin{array}{c}
0 \\
\operatorname{Hom}_{A}\left(M, Y^{\prime}\right)
\end{array}\right] \longrightarrow 0
$$

à l'origine de la suite exacte longue 3.18: on utilise alors le Lemme 1 et le Lemme 2 (bis). 
Pour les versions "homologiques" de la suite exacte longue de MayerVietoris, nous nous donnons un $T$-module (à gauche) et un $T$-module à droite:

$$
f: M \otimes_{B} X \longrightarrow Y \quad \text { et } \quad f^{\prime}: Y^{\prime} \otimes_{A} M \longrightarrow X^{\prime} .
$$

3.21. Lemme 3. Nous avons des isomorphismes canoniques:

$$
\begin{aligned}
& \tilde{s}_{*}: \operatorname{Tor}_{*}^{T}\left(\left[0 X^{\prime}\right],[f]\right) \cong \operatorname{Tor}_{*}^{B}\left(X^{\prime}, X\right) \quad \text { et } \\
& \tilde{b}_{*}: \operatorname{Tor}_{*}^{T}\left(\left[f^{\prime}\right],\left[\begin{array}{l}
Y \\
0
\end{array}\right]\right) \cong \operatorname{Tor}_{*}^{A}\left(Y^{\prime}, Y\right) .
\end{aligned}
$$

Preuve. Nous avons un isomorphisme de $B \otimes T^{o}$-modules: $\left[0 X^{\prime}\right] \cong$ $X^{\prime} \otimes_{B}\left[\begin{array}{ll}0 & B\end{array}\right]$, où $[0 B]$ est $B$-plat et $T^{o}$-plat. Donc $\operatorname{Tor}_{*}^{T}\left(X^{\prime} \otimes_{B}\left[\begin{array}{ll}0 & B\end{array}\right],[f]\right) \cong$ $\operatorname{Tor}_{*}^{B}\left(X^{\prime},[0 B] \otimes_{T}[f]\right)(3.2)$ et, avec 2.13, nous obtenons le premier isomorphisme de 3.22. Le second se démontre pareillement. dué:

3.23. Lemme 4. Si $\operatorname{Tor}_{* \geq 1}^{A}\left(Y^{\prime}, M\right)=0$, nous avons un isomorphisme gra-

$$
\tilde{b}_{*}: \operatorname{Tor}_{*}^{T}\left(\left[1_{Y^{\prime} \otimes_{A} M}\right],[f]\right) \cong \operatorname{Tor}_{*}^{A}\left(Y^{\prime}, Y\right)
$$

Preuve. Nous avons $\operatorname{Tor}_{*}^{T}\left(Y^{\prime} \otimes_{A}[A M],[f]\right) \cong \operatorname{Tor}_{*}^{A}\left(Y^{\prime},\left[\begin{array}{ll}A & M\end{array}\right] \otimes_{T}\right.$ $[f])$ d'après $3.2\left([A M]\right.$ est $T^{o}$-plat et $\left.\operatorname{Tor}_{* \geq 1}^{A}\left(Y^{\prime}, M\right)=0\right)$. Il reste à utiliser l'isomorphisme $\left[1_{Y^{\prime} \otimes_{A} M}\right] \cong Y^{\prime} \otimes_{A}[A M]$ (de $T^{o}$-modules) et l'isomorphisme $[A M] \otimes_{T}[f] \cong Y(2.13)$ pour retrouver 3.24.

Pour une (idée de) démonstration plus conceptuelle de ces lemmes, nous renvoyons à 3.6 .

3.25. Lemme 4 (bis). Si $\operatorname{Tor}_{* \geq 1}^{B}(M, X)=0$, nous avons un isomorphisme gradué:

$$
\tilde{s}_{*}: \operatorname{Tor}_{*}^{T}\left(\left[f^{\prime}\right],\left[1_{M \otimes_{A} X}\right]\right) \cong \operatorname{Tor}_{*}^{B}\left(X^{\prime}, X\right) .
$$

Preuve. Avec l'isomorphisme d'algèbres 2.1, nous sommes dans la situation du Lemme 4, mutatis mutandis.

3.27. THÉORÈME 2. Si $\operatorname{Tor}_{* \geq 1}^{A}\left(Y^{\prime}, M\right)=0$, nous avons une suite exacte longue

$$
\begin{aligned}
\cdots \longrightarrow & \operatorname{Tor}_{*}^{B}\left(Y^{\prime} \otimes_{A} M, X\right) \\
& \longrightarrow \operatorname{Tor}_{*}^{A}\left(Y^{\prime}, Y\right) \oplus \operatorname{Tor}_{*}^{B}\left(X^{\prime}, X\right) \stackrel{\tilde{b}_{*}+\tilde{s}_{*}}{\longrightarrow} \operatorname{Tor}_{*}^{T}\left(\left[f^{\prime}\right],[f]\right) \longrightarrow \cdots
\end{aligned}
$$

où le morphisme $\operatorname{Tor}_{*}^{B}\left(Y^{\prime} \otimes_{A} M, X\right) \longrightarrow \operatorname{Tor}_{*}^{B}\left(X^{\prime}, X\right)$ est induit par $f^{\prime}$. 
Preuve. La suite exacte de $T$-modules $0 \longrightarrow\left[\begin{array}{c}M \otimes_{B} X^{\prime} \\ 0\end{array}\right] \longrightarrow\left[1_{M \otimes_{B} X^{\prime}}\right] \oplus$ $\left[\begin{array}{c}Y^{\prime} \\ 0\end{array}\right] \longrightarrow\left[f^{\prime}\right] \longrightarrow 0$ (cf. 3.14) induit une suite exacte longue pour le foncteur $\operatorname{Tor}_{*}^{T}(.,[f])$. Il reste à appliquer les Lemmes 3 et 4 pour trouver l'écriture 3.28.

3.29. THÉORÈME 2 (bis). Si $\operatorname{Tor}_{* \geq 1}^{B}(M, X)=0$, nous avons une suite exacte longue

$$
\begin{aligned}
\cdots \longrightarrow & \operatorname{Tor}_{*}^{A}\left(Y^{\prime}, M \otimes_{B} X\right) \\
& \longrightarrow \operatorname{Tor}_{*}^{A}\left(Y^{\prime}, Y\right) \oplus \operatorname{Tor}_{*}^{B}\left(X^{\prime}, X\right) \stackrel{\tilde{b}_{*}+\tilde{s}_{*}}{\longrightarrow} \operatorname{Tor}_{*}^{T}\left(\left[f^{\prime}\right],[f]\right) \longrightarrow \cdots
\end{aligned}
$$

où le morphisme $\operatorname{Tor}_{*}^{A}\left(Y^{\prime}, M \otimes_{B} X\right) \longrightarrow \operatorname{Tor}_{*}^{A}\left(Y^{\prime}, Y\right)$ est induit par $f$.

Preuve. On se ramène au Théorème 2 à l'aide de l'isomorphisme d'algèbres 2.1 .

\section{4. (co-)Homologie de Hochschild}

Dans cette section, nous montrons que les suites exactes longues de Happel dérivent directement de celles de Palmér-Roos. Il est à noter que le Théorème 1.1 de [2] ne suffit pas: nous avons besoin de notre Théorème 1 dans toute sa globalité. Le Corollaire 2 est l'un de nos résultats principaux: il généralise [3, 1.3.7 Corollaire].

Une algèbre qui est un $K$-module projectif est dite projective.

Soient trois algèbres $A, B$ et $C$ supposées projectives et soient un $(A, B)$ bimodule $X$ et un $(B, C)$-bimodule $Y$ tels que $\operatorname{Tor}_{* \geq 1}^{B}(X, Y)=0$. Si $Z$ est un $(A, C)$-bimodule tel que $\operatorname{Ext}_{C^{o}}^{* \geq 1}(Y, Z)=0$, nous avons un isomorphisme gradué ([6], (4) p.346):

$$
\operatorname{Ext}_{A \otimes C^{o}}^{*}\left(X \otimes_{B} Y, Z\right) \cong \operatorname{Ext}_{A \otimes B^{o}}^{*}\left(X, \operatorname{Hom}_{C^{o}}(Y, Z)\right) .
$$

Dualement, si $Z^{\prime}$ est un $(C, A)$-bimodule tel que $\operatorname{Tor}_{* \geq 1}^{C}\left(Y, Z^{\prime}\right)=0$, nous avons:

$$
\operatorname{Tor}_{*}^{C \otimes A^{o}}\left(X \otimes_{B} Y, Z^{\prime}\right) \cong \operatorname{Tor}_{*}^{B \otimes A^{o}}\left(X, Y \otimes_{C} Z^{\prime}\right) \quad \text { ([6], (4a) p. 347). }
$$

L'homologie et la cohomologie de Hochschild de toute algèbre projective $T$ à coefficients dans un $T$-bimodule $\Lambda$, sont définies par les modules gradués:

$$
H_{*}(T, \Lambda)=\operatorname{Tor}_{*}^{T \otimes T^{o}}(T, \Lambda) \quad \text { et } \quad H^{*}(T, \Lambda)=\operatorname{Ext}_{T \otimes T^{o}}^{*}(T, \Lambda) .
$$

Par définition, $H H_{*}(T)=H_{*}(T, T)$ et $H H^{*}(T)$ est l'algèbre ${ }^{(\dagger)} H^{*}(T, T)$ (produit de Yoneda). Dans le cas d'une algèbre triangulaire 1.2, un $T$-bimodule

\footnotetext{
${ }^{(\dagger)}$ attention: dans [18], $H H^{*}(T)$ signifie $H^{*}\left(T, \operatorname{Hom}_{K}(T, K)\right)$
} 
$\Lambda$ est défini par des bimodules $\left\{X_{1}, X_{2}, Y_{1}, Y_{2}\right\}$ et des morphismes de bimodules $M \otimes_{B} X_{1} \stackrel{f_{1}}{\longrightarrow} Y_{1}, M \otimes_{B} X_{2} \stackrel{f_{2}}{\longrightarrow} Y_{2}, X_{1} \otimes_{A} M \stackrel{\varphi_{X}}{\longrightarrow} X_{2}$ et $Y_{1} \otimes_{A} M \stackrel{\varphi_{Y}}{\longrightarrow} Y_{2}$, faisant commuter le diagramme:

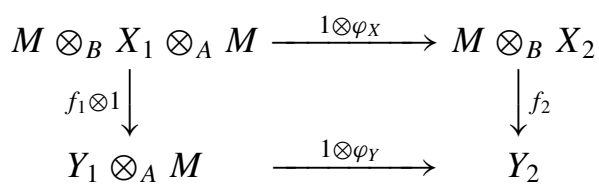

Nous adoptons, à l'instar des $T$-modules, une écriture matricielle: $\Lambda=\left[\begin{array}{ll}Y_{1} & Y_{2} \\ X_{1} & X_{2}\end{array}\right]$ (compatible avec celle du $T$-bimodule $T=\left[\begin{array}{cc}A & M \\ 0 & B\end{array}\right]$ ).

Le résultat suivant est "la suite exacte longue de Happel" (cohomologique): nous supposons dorénavant que $A, B$ et $M$ sont $K$-projectifs.

4.5. THÉORÈME 3. Avec les notations de 4.4, nous avons une suite exacte longue:

$$
\begin{aligned}
& \cdots \longrightarrow \operatorname{Ext}_{A \otimes B^{o}}^{*-1}\left(M, Y_{2}\right) \longrightarrow H^{*}(T, \Lambda) \\
& \stackrel{b^{*}}{s^{*}} \begin{array}{l}
H^{*}\left(A, Y_{1}\right) \\
H^{*}\left(B, X_{2}\right)
\end{array} \longrightarrow \operatorname{Ext}_{A \otimes B^{o}}^{*}\left(M, Y_{2}\right) \longrightarrow \cdots
\end{aligned}
$$

De plus, $H^{*}(T, \Lambda)$ ne dépend pas de $X_{1}$.

4.7. Preuve. Nous avons une structure triangulaire sur $T \otimes T^{o}$ :

$$
T \otimes T^{o} \cong\left[\begin{array}{cc}
T_{A} & T_{M} \\
0 & T_{B}
\end{array}\right]: \quad T_{A}=A \otimes T^{o}, T_{B}=B \otimes T^{o}, T_{M}=M \otimes T .
$$

De là, une écriture matricielle $\Lambda=\left[\begin{array}{l}Y \\ X\end{array}\right]$, avec $Y=\left[\begin{array}{ll}Y_{1} & Y_{2}\end{array}\right]$ et $X=\left[\begin{array}{ll}X_{1} & X_{2}\end{array}\right]$. Remarquons qu'il existe d'autres décompositions triangulaires, telle celle $T \otimes$ $T^{o} \cong\left[\begin{array}{cc}A^{\natural} & M^{\natural} \\ 0 & B^{\natural}\end{array}\right]$, avec $A^{\natural}=\left[\begin{array}{cc}T_{A} & \tilde{M} \otimes B \\ 0 & B \otimes B^{o}\end{array}\right]\left(\tilde{M}=\left[\begin{array}{c}M \\ 0\end{array}\right]\right)$ et $B^{\natural}=B \otimes A^{o}$ (inutile d'expliciter $M^{\natural}$ ): cette fois, l'écriture matricielle du $T \otimes T^{o}$-module $\Lambda$ est $\left[\begin{array}{c}\hat{\Lambda} \\ X_{1}\end{array}\right]$, où $\hat{\Lambda}=\left[\begin{array}{c}Y \\ X_{2}\end{array}\right]$; en particulier $T=\left[\begin{array}{c}\hat{T} \\ 0\end{array}\right]$ (inutile d'expliciter $\hat{T}$ ) et, d'après 3.5, nous avons $\operatorname{Ext}_{T \otimes T^{o}}^{*}(T, \Lambda) \cong \operatorname{Ext}_{A^{\natural}}^{*}(\hat{T}, \hat{\Lambda})$, i.e. $H^{*}(T, \Lambda)$ ne dépend pas de $X_{1}$. Retournons à l'écriture triangulaire 4.8. D'après 4.2 , nous avons:

$$
\begin{aligned}
\operatorname{Tor}_{* \geq 1}^{B \otimes T^{o}}\left(M \otimes_{K} T,\left[\begin{array}{ll}
0 & B
\end{array}\right]\right) & \cong \operatorname{Tor}_{* \geq 1}^{K \otimes B}\left(M, T \otimes_{T^{o}}\left[\begin{array}{ll}
0 & B
\end{array}\right]\right) \\
& \cong \operatorname{Tor}_{* \geq 1}^{B}(M, B)=0,
\end{aligned}
$$

soit $\operatorname{Tor}_{* \geq 1}^{T_{B}}\left(T_{M},\left[\begin{array}{ll}0 & B\end{array}\right]\right)=0$. Nous avons donc la suite exacte longue du 
Théorème 1:

$$
\begin{aligned}
& \cdots \longrightarrow \operatorname{Ext}_{T \otimes T^{o}}^{*}(T, \Lambda) \\
& \stackrel{b^{b^{*}}}{\longrightarrow} \operatorname{Ext}_{T_{A}}^{*}\left(\left[\begin{array}{ll}
A & M
\end{array}\right], Y\right) \\
& \operatorname{Ext}_{T_{B}}^{*}\left(\left[\begin{array}{ll}
0 & B
\end{array}\right], X\right)
\end{aligned} \longrightarrow \operatorname{Ext}_{T_{A}}^{*}\left(T_{M} \otimes_{T_{B}}\left[\begin{array}{ll}
0 & B
\end{array}\right], Y\right) \longrightarrow \cdots
$$

Par associativité, nous avons des isomorphismes de $T_{A}$-modules:

$$
\begin{aligned}
T_{M} \otimes_{T_{B}}\left[\begin{array}{ll}
0 & B
\end{array}\right] & \cong\left(M \otimes_{K} T\right) \otimes_{B \otimes T^{o}}\left[\begin{array}{ll}
0 & B
\end{array}\right] \\
& \cong M \otimes_{B}\left(T \otimes_{T^{o}}\left[\begin{array}{ll}
0 & B
\end{array}\right]\right) \\
& \cong\left[\begin{array}{ll}
0 & M
\end{array}\right] .
\end{aligned}
$$

Donc, d'après 3.5, nous avons $\operatorname{Ext}_{T_{A}}^{*}\left(T_{M} \otimes_{T_{B}}[0 B], Y\right) \cong \operatorname{Ext}_{A \otimes B^{o}}^{*}(M, Y)$ et, pour une raison identique, $\operatorname{Ext}_{T_{B}}^{*}\left(\left[\begin{array}{ll}0 & B\end{array}\right], X\right) \cong \operatorname{Ext}_{B \otimes B^{o}}^{*}\left(B, X_{2}\right)$. D'après 4.2 ( $A$ est $K$-projectif):

$$
\begin{aligned}
\operatorname{Tor}_{* \geq 1}^{A \otimes A^{o}}\left(A \otimes_{K} M, A\right) & \cong \operatorname{Tor}_{* \geq 1}^{K \otimes A}\left(A, M \otimes_{A^{o}} A\right) \\
& \cong \operatorname{Tor}_{* \geq 1}^{A}(A, M)=0 .
\end{aligned}
$$

Il existe donc une suite exacte longue de Mayer-Vietoris (Théorème 1)

$$
\begin{aligned}
\cdots \longrightarrow \operatorname{Ext}_{T_{A}}^{*}\left(\left[\begin{array}{ll}
{[A} & M
\end{array}\right],\left[\begin{array}{ll}
Y_{1} & Y_{2}
\end{array}\right]\right) \\
\qquad \begin{array}{c}
\operatorname{Ext}_{A \otimes B^{o}}^{*}\left(M, Y_{2}\right) \\
\operatorname{Ext}_{A \otimes A^{o}}^{*}\left(A, Y_{1}\right)
\end{array} \longrightarrow \operatorname{Ext}_{A \otimes B^{o}}^{*}\left(M, Y_{2}\right) \longrightarrow \cdots
\end{aligned}
$$

qui fournit immédiatement un isomorphisme:

$$
\operatorname{Ext}_{T_{A}}^{*}\left([A M],\left[Y_{1} Y_{2}\right]\right) \cong \operatorname{Ext}_{A \otimes A^{o}}^{*}\left(A, Y_{1}\right)
$$

Ce qui achève la preuve du Théorème 3 .

4.15. Corollarie 1. Supposons que $K$ soit un corps et que $T$ soit de dimension finie. Si le quotient de $A$ et $B$ par le radical est $K$-séparable (par exemple: $\operatorname{car}(K)=0$ ou $K$ algébriquement clos), alors gldim $\left[\begin{array}{cc}A & M \\ 0 & B\end{array}\right]=$ $\max \left\{\operatorname{gldim}(A), \operatorname{gldim}(B), 1+\operatorname{pdim}_{A \otimes B^{o}}(M)\right\}$.

Preuve. Avec ces hypothèses, nous avons gldim $T=\operatorname{pdim}_{T \otimes T^{o}} T$ d'après [1]. Et il suffit d'utiliser la suite exacte longue 4.6 pour conclure.

Le résultat suivant est une généralisation de [3, 1.3.7 Corollaire].

4.16. Corollarie 2. Soit $M$ un A-module $K$-projectif tel que $B=\left(\operatorname{End}_{A}\right.$ $M)^{o}$ soit aussi $K$-projectif (par exemple: $K$ semi-simple) et que $\operatorname{Ext}_{A}^{* \geq 1}(M, M)$ $=0$. Nous avons un isomorphisme d'algèbres graduées:

$$
b^{*}: H H^{*}\left[\begin{array}{ll}
A & M \\
0 & B
\end{array}\right] \stackrel{\sim}{\longrightarrow} H H^{*}(A) .
$$


Nous déduisons aussi de ce corollaire un isomorphisme $H H^{*}(B) \cong$ $\operatorname{Ext}_{A \otimes B^{o}}^{*}(M, M)$. Par ailleurs, il existe aussi un résultat symétrique si l'on dispose d'un $B^{o}$-module $M$ tel que $\operatorname{Ext}_{B^{o}}^{* \geq 1}(M, M)=0$.

Preuve. Nous utilisons les notations précédentes. Avec 3.5, nous avons un isomorphisme:

$$
\operatorname{Ext}_{T^{e}}^{*}(T, \Lambda) \cong \operatorname{Ext}_{T^{\prime}}^{*}\left(\left[\begin{array}{c}
M^{\prime} \\
B
\end{array}\right],\left[\begin{array}{c}
Y \\
X_{2}
\end{array}\right]\right),
$$

où $T^{\prime}=\left[\begin{array}{cc}T_{A} & M_{B} \\ 0 & B \otimes B^{0}\end{array}\right], M_{B}=[0 M \otimes B]$ et $M^{\prime}=[A M]$. Toujours avec 3.5, la suite exacte courte de $T^{\prime}$-modules: $0 \rightarrow\left[\begin{array}{c}M^{\prime} \\ 0\end{array}\right] \rightarrow\left[\begin{array}{c}M^{\prime} \\ B\end{array}\right] \rightarrow\left[\begin{array}{c}0 \\ B\end{array}\right] \rightarrow 0$ induit une suite exacte longue:

$$
\begin{aligned}
\cdots \longrightarrow \operatorname{Ext}_{\left(T_{B}\right)^{o}}^{*} & \left(\left[\begin{array}{l}
0 \\
B
\end{array}\right],\left[\begin{array}{c}
Y \\
X_{2}
\end{array}\right]\right) \\
& \longrightarrow \operatorname{Ext}_{T^{\prime}}^{*}\left(\left[\begin{array}{c}
M^{\prime} \\
B
\end{array}\right],\left[\begin{array}{c}
Y \\
X_{2}
\end{array}\right]\right) \longrightarrow \operatorname{Ext}_{T_{A}}^{*}\left(M^{\prime}, Y\right) \longrightarrow \cdots
\end{aligned}
$$

où l'on a utilisé l'autre structure triangulaire $T^{\prime} \cong\left[\begin{array}{cc}\left(T_{B}\right)^{o} & { }_{A} M \\ 0 & A \otimes A^{o}\end{array}\right]$, avec ${ }_{A} M=$ $\left[\begin{array}{c}A \otimes M \\ 0\end{array}\right]$. Nous connaissons déjà l'isomorphisme $\operatorname{Ext}_{T_{A}}^{*}\left(M^{\prime}, Y\right) \cong \operatorname{Ext}_{A \otimes A^{o}}^{*}(A$, $\left.Y_{1}\right)$ (4.14). Un argument identique prouve que nous avons aussi $\operatorname{Ext}_{\left(T_{B}\right)^{\circ}}^{*}\left(\left[\begin{array}{c}M \\ B\end{array}\right]\right.$, $\left.\left[\begin{array}{l}Y_{2} \\ X_{2}\end{array}\right]\right) \cong \operatorname{Ext}_{B \otimes B^{o}}^{*}\left(B, X_{2}\right)$. La suite exacte longue, déduite de la suite exacte courte $0 \longrightarrow\left[\begin{array}{c}M \\ 0\end{array}\right] \longrightarrow\left[\begin{array}{c}M \\ B\end{array}\right] \longrightarrow\left[\begin{array}{l}0 \\ B\end{array}\right] \longrightarrow 0$,

$$
\begin{aligned}
\cdots \longrightarrow \operatorname{Ext}_{\left(T_{B}\right)^{o}}^{*}\left(\left[\begin{array}{l}
0 \\
B
\end{array}\right],\left[\begin{array}{c}
Y_{2} \\
X_{2}
\end{array}\right]\right) \longrightarrow \operatorname{Ext}_{\left(T_{B}\right)^{o}}^{*}\left(\left[\begin{array}{c}
M \\
B
\end{array}\right],\left[\begin{array}{c}
Y_{2} \\
X_{2}
\end{array}\right]\right) & \\
& \longrightarrow \operatorname{Ext}_{A \otimes B^{o}}^{*}\left(M, Y_{2}\right) \longrightarrow \cdots
\end{aligned}
$$

se récrit dans le cas particulier où $\Lambda=T$ :

$$
\begin{aligned}
\cdots \longrightarrow \operatorname{Ext}_{\left(T_{B}\right)^{o}}^{*}\left(\left[\begin{array}{l}
0 \\
B
\end{array}\right],\left[\begin{array}{l}
Y_{2} \\
X_{2}
\end{array}\right]\right) \longrightarrow & H^{*}\left(B, X_{2}\right) \\
& \stackrel{H^{*}\left(B, \tilde{f}_{2}\right)}{\longrightarrow} H^{*}\left(B, \operatorname{Hom}_{A}\left(M, Y_{2}\right)\right) \longrightarrow \cdots
\end{aligned}
$$

puisque $\operatorname{Ext}_{A \otimes B^{o}}^{*}(M, M) \cong \operatorname{Ext}_{B \otimes B^{o}}^{*}\left(B, \operatorname{Hom}_{A}(M, M)\right)$ d'après 4.1: c'est ici qu'intervient l'hypothèse $E x t_{A}^{* \geq 1}(M, M)=0$. Nous en déduisons l'annulation $\operatorname{Ext}_{\left(T_{B}\right)^{o}}^{*}\left(\left[\begin{array}{l}0 \\ B\end{array}\right],\left[\begin{array}{l}Y_{2} \\ X_{2}\end{array}\right]\right)=0$ et la suite exacte 4.18 se réduit à l'isomorphisme 4.17.

4.19. Exemple 1 ([11, preuve du Théorème 7.3]). Posons $A=\left[\begin{array}{ll}B & B \\ 0 & B\end{array}\right]$ (pour une certaine algèbre $K$-projective $B)$ et $M=\left[\begin{array}{l}0 \\ B\end{array}\right]$. D'une part $\operatorname{Ext}_{A}^{* \geq 1}(M, M) \cong$ $\operatorname{Ext}_{B}^{* \geq 1}(B, B)=0$ (3.5) et, d'autre part, $\left(\operatorname{End}_{A} M\right)^{o} \cong B$. Avec le Corollaire 2, nous obtenons:

$$
H H^{*}\left[\begin{array}{ccc}
B & B & 0 \\
0 & B & B \\
0 & 0 & B
\end{array}\right] \cong H H^{*}\left[\begin{array}{ll}
B & B \\
0 & B
\end{array}\right] \cong H H^{*}(B) \quad \text { (cf. 1.5). }
$$


Notons que $M$ n'est pas $A$-projectif. Si $B=K$ est un corps, alors $M$ est $A$-injectif puisque $M=\operatorname{Hom}_{K}([0 K], K)$ et que le $A^{o}$-module $[0 K]$ est projectif.

Le résultat suivant est "la suite exacte longue de Happel" (homologique).

4.21. ThÉORÈME 3 (bis). Avec les notations de 4.4, nous avons une suite exacte longue:

$$
\begin{aligned}
\cdots \longrightarrow \operatorname{Tor}_{*}^{B \otimes A^{o}}\left(M, X_{1}\right) & \longrightarrow \begin{array}{l}
H_{*}\left(A, Y_{1}\right) \\
H_{*}\left(B, X_{2}\right)
\end{array} \\
\stackrel{\tilde{\tilde{b}}_{*}+\tilde{s}_{*}}{\longrightarrow} & H_{*}(T, \Lambda) \longrightarrow \operatorname{Tor}_{*-1}^{B \otimes A^{o}}\left(M, X_{1}\right) \longrightarrow \cdots
\end{aligned}
$$

De plus, $H_{*}(T, \Lambda)$ ne dépend pas de $Y_{2}$.

En particulier: nous retrouvons 1.3 avec $\Lambda=T$ puisque, auquel cas, nous avons $X_{1}=0$.

Preuve. La preuve est duale de celle du Théorème 3 , dont nous reprenons les notations: $T \otimes T^{o} \cong\left[\begin{array}{cc}T_{A} & T_{M} \\ 0 & T_{B}\end{array}\right]$ et le $T \otimes T^{o}$-module à droite $T$ admet une écriture matricielle $\left[Y^{\prime} X^{\prime}\right]$, avec $Y^{\prime}=\left[\begin{array}{l}A \\ 0\end{array}\right]$ et $X^{\prime}=\left[\begin{array}{c}M \\ B\end{array}\right]=\left[1_{M}\right]$. D'après 4.2, nous avons les isomorphismes:

$$
\operatorname{Tor}_{* \geq 1}^{T_{A}}\left(Y^{\prime}, M \otimes T\right)=\operatorname{Tor}_{* \geq 1}^{A \otimes T^{o}}\left(T \otimes_{T} Y^{\prime}, M \otimes_{K} T\right) \cong \operatorname{Tor}_{* \geq 1}^{A \otimes K}(A, M)=0 .
$$

Nous en déduisons une suite exacte longue de Mayer-Vietoris (Théorème 2):

$$
\begin{aligned}
& \cdots \longrightarrow \operatorname{Tor}_{*+1}^{T \otimes T^{o}}(T, \Lambda) \longrightarrow \operatorname{Tor}_{*}^{T_{B}}\left(Y^{\prime} \otimes_{T_{A}} T_{M}, X\right) \\
& \longrightarrow \begin{array}{c}
\operatorname{Tor}_{*}^{T_{A}}\left(X^{\prime}, X\right) \\
\operatorname{Tor}_{*}^{T_{B}}\left(Y^{\prime}, Y\right)
\end{array} \stackrel{\tilde{b}_{*}+\tilde{s}_{*}}{\longrightarrow} \operatorname{Tor}_{*}^{T \otimes T^{o}}(T, \Lambda) \longrightarrow \cdots
\end{aligned}
$$

Avec les structures triangulaires $T_{A} \cong\left[\begin{array}{cc}A \otimes B^{o} & A \otimes M \\ 0 & A \otimes A^{o}\end{array}\right]$ et $T_{B} \cong\left[\begin{array}{ccc}B \otimes B^{o} & B \otimes M \\ 0 & B \otimes A^{o}\end{array}\right]$, nous avons des isomorphismes $\operatorname{Tor}_{*}^{T_{A}}\left(Y^{\prime}, Y\right) \cong \operatorname{Tor}_{*}^{A \otimes A^{o}}\left(A, Y_{1}\right) \cong H_{*}\left(A, Y_{1}\right)$ et $\operatorname{Tor}_{*}^{T_{B}}\left(Y^{\prime} \otimes_{T_{A}} T_{M}, X\right) \cong \operatorname{Tor}_{*}^{T_{B}}\left(Y^{\prime} \otimes_{A} M, X\right) \cong \operatorname{Tor}_{*}^{B \otimes A^{o}}\left(M, X_{1}\right)$ (cf. 3.22). Puisque $X^{\prime}$ est $T$-projectif, nous avons:

$$
\operatorname{Tor}_{*}^{B \otimes T^{o}}\left(B \otimes_{B} X^{\prime}, X\right) \cong \operatorname{Tor}_{*}^{B \otimes B^{o}}\left(B, X^{\prime} \otimes_{T^{o}} X\right) \cong \operatorname{Tor}_{*}^{B \otimes B^{o}}\left(B, X^{\prime} \otimes_{T^{o}} X\right)
$$

(cf. 4.2), où $X^{\prime} \otimes_{T^{o}} X \cong X_{2}$ (2.13); de là $\operatorname{Tor}_{*}^{T_{B}}\left(X^{\prime}, X\right) \cong H_{*}\left(B, X_{2}\right)$ et la suite exacte longue 4.22 est celle 4.23. L'indépendance de $H_{*}(T, \Lambda)$ envers $Y_{2}$ peut s'obtenir en introduisant une décomposition triangulaire ad hoc: $T \otimes T^{o} \cong$

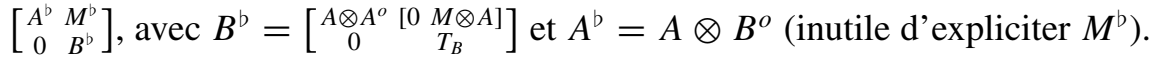


Ainsi nous avons l'écriture matricielle: $\Lambda=\left[\begin{array}{c}Y_{2} \\ \check{\Lambda}\end{array}\right]$, où $\check{\Lambda}=\left[\begin{array}{c}Y_{1} \\ X\end{array}\right]$. Pour le $T \otimes T^{o}$ module à droite $T$, nous obtenons [0 $\check{T}]$ (inutile d'expliciter $\breve{T}$ ) et, d'après 3.22, $\operatorname{Tor}_{*}^{T \otimes T^{o}}(T, \Lambda) \cong \operatorname{Tor}_{*}^{B^{b}}(\check{T}, \check{\Lambda})$ ne dépend pas de $Y_{2}$.

\section{Suite Exacte Longue de Mayer-Vietoris}

Dans cette section, nous montrons combien les techniques "triangulaires" développées dans $\$ \mathbf{3}$ et $\$ \mathbf{4}$ concernent très pertinemment les posets. Même si nous nous focalisons sur les résultats cohomologiques, toutes nos considérations se dualisent immédiatement et l'on saura en déduire facilement des versions homologiques.

Un ensemble ordonné fini $P=(P, \geq)$ est brièvement appelé un "poset" (leurs morphismes sont donc les applications croissantes); nous notons $P^{o}$, le poset "opposé" $(P, \leq)$. Un sous-poset de $P$ est un sous-ensemble $Q \subset P$ muni de la relation d'ordre induite; il est dit terminal (resp. initial) si:

$$
\forall p \in P, \forall q \in Q, \quad p \geq q \Longrightarrow p \in Q \quad(\text { resp. } q \geq p \Longrightarrow p \in Q \text { ). }
$$

On montre facilement que $Q$ est initial dans $P$ si et seulement si son complémentaire $P \backslash Q$ est terminal dans $P$. Pour tout ce qui concerne les propriétés homologiques des posets, nous renvoyons à [21]; un exemple d'application est donné dans [5].

Posons $K^{P}=\operatorname{Hom}\left(K^{(P)}, K\right)$ : c'est un module libre (isomorphe à $\left.K^{(P)}\right)$, de base duale $\left\{x^{*} \mid x \in P\right\}$. Il est facile de voir que le sous-module libre de $\operatorname{End}\left(K^{(P)}\right)=K^{(P)} \otimes K^{P}$ de base $\left\{y \otimes x^{*} \mid y \geq x\right\}$ est une sousalgèbre, appelée algèbre d'incidence de $P$ et notée $K[P]$. Donc $K^{(P)}$ (resp. $K^{P}$ ) est naturellement un $K[P]$-module (resp. un $K[P]^{o}$-module). L'algèbre d'incidence est compatible à certaines opérations:

$$
\begin{aligned}
K[P]^{o} & =K\left[P^{o}\right], \\
K[Q \sqcup P] & \cong K[Q] \times K[P] \quad \text { et } \\
K[Q \times P] & \cong K[Q] \otimes K[P] .
\end{aligned}
$$

Si $Q$ est un sous-poset terminal de $P$, nous avons une structure d'algèbre triangulaire:

$$
K[P] \cong\left[\begin{array}{cc}
K[Q] & Q^{M} \backslash Q \\
0 & K[P \backslash Q]
\end{array}\right],
$$

pour un certain $K[Q] \otimes K[P \backslash Q]^{o}$-sous-module ${ }_{Q} M_{P \backslash Q}$ de $H_{o} m_{K}\left(K^{(P \backslash Q)}\right.$, $\left.K^{(Q)}\right)$. 
5.4. LeMme 5. Pour tout poset $P$, nous avons un isomorphisme d'algèbres graduées:

$$
H H^{*}(K[P]) \cong \operatorname{Ext}_{K[P]}^{*}\left(K^{(P)}, K^{(P)}\right)
$$

Preuve. La démonstration suivante, proposée par le rapporteur, est différente de celle esquissée dans [2] et revient essentiellement à montrer le Théorème $\mathbf{S C}=\mathbf{H C}$ de [14] dans notre cas particulier. Pour cela, rappelons que l'équivalence naturelle de catégories $\mathscr{F} \longmapsto \widehat{\mathscr{F}}$, entre celle des foncteurs en $K$-modules sur $P$ (vu comme une catégorie) et celle des $K[P]$-modules, interprète la cohomologie de Quillen $H^{*}(P, \mathscr{F})=\lim _{\leftarrow}^{*} \mathscr{F}$ (dérivées à droite du foncteur limite), comme un foncteur Ext via l'isomorphisme: $H^{*}(P, \mathscr{F}) \cong$ $\operatorname{Ext}_{K[P]}^{*}\left(K^{(P)}, \widehat{\mathscr{F}}\right)$. Si $\mathscr{F}$ est le foncteur constant égal à $K$, nous avons un isomorphisme de $K[P]$-modules $\widehat{\mathscr{F}} \cong K^{(P)}$ et un isomorphisme d'algèbres graduées: $H^{*}(P, K) \cong \operatorname{Ext}_{K[P]}^{*}\left(K^{(P)}, K^{(P)}\right)$.

Considérons à présent le sous-poset $P^{\#}=\{(y, x) \mid y \geq x\}$ de $P \times P^{o}$ : il est terminal et le module $K[P]$ sur l'anneau $K[P] \otimes K[P]^{o} \cong K[P \times$ $\left.P^{o}\right]$ est isomorphe à $K^{\left(P^{*}\right)}$. Nous en déduisons un isomorphisme d'algèbres graduées $H H^{*}(K[P]) \cong \operatorname{Ext}_{K\left[P^{*}\right]}^{*}\left(K^{\left(P^{*}\right)}, K^{\left(P^{*}\right)}\right)$ (3.5) soit, $H H^{*}(K[P]) \cong$ $H^{*}\left(P^{\#}, K\right)$. Or la première projection canonique induit une équivalence d'homotopie $P^{\#} \longrightarrow P$ : c'est le Théorème $\mathrm{A}$ de Quillen; en effet, pour tout $z \in P$, le poset $\left\{(y, x) \in P^{\#} \mid y \leq z\right\}$ est contractile puisque nous avons les inégalités $(y, x) \leq(z, x) \geq(z, z)$ (cf. [24, 1.5]). De là un isomorphisme $H^{*}\left(P^{\#}, K\right) \longrightarrow H^{*}(P, K)$ et celui 5.5.

Pour deux posets $P$ et $Q$, définissons une relation $\mathscr{R}: P \longrightarrow Q$, comme étant un morphisme $P \longrightarrow \mathscr{P}(Q)$ (parties de $Q$ ordonnées par l'inclusion) tel que: pour tout $x \in P, \mathscr{R}(x)$ est terminal dans $Q$. Nous en déduisons un poset "graphe" $C_{\mathscr{R}}=(Q \sqcup P, \geq)$, en ajoutant aux relations de $P$ et de $Q$, celles: $y \geq x$ si $y \in Q, x \in P$ et $y \in \mathscr{R}(x)$. Clairement, $P$ est un sous-poset initial de $C_{\mathscr{R}}$ et nous avons une écriture triangulaire 5.3 pour $K\left[C_{\mathscr{R}}\right]$. Nous allons traiter deux cas particuliers très intéressants: le "cylindre" de Gerstenhaber et Schack ([13]) et les recouvrements "terminaux" qui mènent à la suite de Mayer-Vietoris.

5.6. Le cylindre. Pour tout morphisme de posets $\varphi: P \longrightarrow Q$, nous avons la relation $\mathscr{R}(x)=\{y \in Q \mid y \geq \varphi(x)\}$ et son graphe $C_{\varphi}=C_{\mathscr{R}}$ (noté cyl $\varphi$ dans [2]), avec

$$
K\left[C_{\varphi}\right] \cong\left(\begin{array}{cc}
K[Q] & K[\varphi] \\
0 & K[P]
\end{array}\right) .
$$


Nous expliciterons le $K[Q] \otimes K[P]^{\circ}$-module $K[\varphi]$ dans la preuve du lemme suivant.

5.8. Lemme 6. Pour tous $K\left[C_{\varphi}\right]$-modules $\Lambda=\left[\begin{array}{l}Y \\ X\end{array}\right], \Lambda^{\prime}=\left[\begin{array}{l}Y^{\prime} \\ X^{\prime}\end{array}\right]$, nous avons une suite exacte:

$$
\begin{aligned}
\cdots \rightarrow \operatorname{Ext}_{K\left[C_{\varphi}\right]}^{*}\left(\Lambda, \Lambda^{\prime}\right) \rightarrow & \operatorname{Ext}_{K[Q]}^{*}\left(Y, Y^{\prime}\right) \\
& \rightarrow \operatorname{Ext}_{K[P]}^{*}\left(X, X^{\prime}\right) \\
&
\end{aligned}
$$

Preuve. C'est la suite exacte longue du Théorème 1 bis (cf. §3): pour l'obtenir, il suffit de vérifier que le $K[Q]$-module $K[\varphi]$ est projectif. Or, par définition,

$$
K[\varphi]=\bigoplus_{y \geq \varphi(x)} K y \otimes x^{*}=\bigoplus_{x \in P} K[Q] \varphi(x) \otimes x^{*} \cong \bigoplus_{y \in \operatorname{Im} \varphi}\left(K[Q] y \otimes y^{*}\right)^{\varphi^{-1} y}
$$

où, pour chaque $y \in Q, y \otimes y^{*}$ est un idempotent de $K[Q]$ générant un $K[Q]$-module projectif.

5.10. THÉORÈME 4. Pour tout $K\left[C_{\varphi}\right]$-module $\Lambda=\left[\begin{array}{l}Y \\ X\end{array}\right]$, nous avons un isomorphisme gradué:

$$
b^{*}: \operatorname{Ext}_{K\left[C_{\varphi}\right]}^{*}\left(\left[\begin{array}{l}
Y \\
X
\end{array}\right], K^{\left(C_{\varphi}\right)}\right) \cong \operatorname{Ext}_{K[Q]}^{*}\left(Y, K^{(Q)}\right) .
$$

En particulier: $\operatorname{idim}_{K\left[C_{\varphi}\right]} K^{\left(C_{\varphi}\right)}=\operatorname{idim}_{K[Q]} K^{(Q)}$.

Preuve. Supposons que le $K\left[C_{\varphi}\right]$-module $\Lambda$ soit décrit par un morphisme de $K[Q]$-modules $f: K[\varphi] \otimes_{K[P]} X \rightarrow Y$. Le morphisme $\operatorname{Ext}_{K[P]}^{*}\left(X, X^{\prime}\right) \rightarrow$ $\operatorname{Ext}_{K[P]}^{*}\left(X, \operatorname{Hom}_{K[Q]}\left(K[\varphi], Y^{\prime}\right)\right)$ du Lemme 6 est induit (d'après le Théorème 1 bis) par $\tilde{f}^{\prime}: X^{\prime} \longrightarrow \operatorname{Hom}_{K[Q]}\left(K[\varphi], Y^{\prime}\right)$; ici $X^{\prime}=K^{(P)}, Y^{\prime}=K^{(Q)}$ et $f^{\prime}: K[\varphi] \otimes_{K[P]} K^{(P)} \longrightarrow K^{(Q)}$ est défini par $f^{\prime}(m \otimes p)=\delta_{p}^{x} y$ si $m=y \otimes x^{*}$ (cf. 5.9). Or nous avons les isomorphismes:

$$
\begin{aligned}
\operatorname{Hom}_{K[Q]}\left(K[\varphi], K^{(Q)}\right) & \cong \prod_{x \in P} \operatorname{Hom}_{K[Q]}\left(K[Q] \varphi(x) \otimes x^{*}, K^{(Q)}\right) \\
& \cong \prod_{x \in P} \varphi(x) K^{(Q)} \cong K^{(P)} .
\end{aligned}
$$

Donc $\tilde{f}^{\prime}$ est un isomorphisme et, nécessairement, la suite exacte longue du Lemme 6 se réduit à l'isomorphisme gradué 5.11.

Choisissant $\Lambda=K^{\left(C_{\varphi}\right)}$ dans 5.11, nous obtenons:

$$
\operatorname{Ext}_{K\left[C_{\varphi}\right]}^{*}\left(K^{\left(C_{\varphi}\right)}, K^{\left(C_{\varphi}\right)}\right)=\operatorname{Ext}_{K[Q]}^{*}\left(K^{(Q)}, K^{(Q)}\right),
$$


soit le Théorème 3.1 de [2] d'après le Lemme 5:

$$
H H^{*}\left(K\left[C_{\varphi}\right]\right) \cong H H^{*}(K[Q]) .
$$

Comme dans la preuve du Lemme 5, remarquons (avec le rapporteur) que 5.13 peut aussi s'obtenir à l'aide du Théorème $\mathrm{A}$ de Quillen qui prouve que le morphisme $C_{\varphi} \longrightarrow Y, y \longmapsto y$ et $x \longmapsto \varphi(x)$, est une équivalence d'homotopie; on conclut avec le Théorème $\mathbf{S C}=\mathbf{H C}$.

5.14. Recouvrements. Dans cette sous-section nous rattachons la suite exacte longue de Palmér-Roos et, incidemment, celle de Happel, à la suite exacte longue suivante.

5.15. Proposition 4. Si $Q=Q_{1} \cup Q_{2}$ est un recouvrement d'un poset $Q$, par deux sous-posets terminaux, nous avons une suite exacte longue:

$$
\begin{aligned}
\cdots \longrightarrow H H^{*}(K[Q]) \longrightarrow H H^{*}\left(K\left[Q_{1}\right]\right) \times H H^{*}\left(K\left[Q_{2}\right]\right) & \\
& \longrightarrow H H^{*}\left(K\left[Q_{1} \cap Q_{2}\right]\right) \longrightarrow \cdots
\end{aligned}
$$

Il s'agit de la suite exacte longue (cohomologique) de Mayer-Vietoris chère aux topologues (cf. [9]). Pour s'en persuader, on peut utiliser l'isomorphisme $H^{*}(\Sigma, K) \cong H H^{*}(K[Q])$ (Théorème $\mathbf{S C}=\mathbf{H C}$ : l'algèbre $K[Q]$ est notée $\mathbf{k}_{\Sigma}$ ! dans [14]), où $H^{*}(\Sigma, K)$ est la $K$-cohomologie simpliciale d'un ensemble simplicial $\Sigma$, naturellement attaché à $Q$. Nous pouvons aussi appliquer le bifoncteur $\operatorname{Ext}_{K[Q]}^{*}$ au module $K^{(Q)}$ et à la suite exacte courte

$$
0 \longrightarrow K^{\left(Q_{1} \cap Q_{2}\right)} \longrightarrow K^{\left(Q_{1}\right)} \oplus K^{\left(Q_{2}\right)} \longrightarrow K^{(Q)} \longrightarrow 0 .
$$

Le Lemme $1 \mathrm{~s}$ 'applique plusieurs fois, transformant la suite exacte longue en une autre:

$$
\begin{aligned}
& \cdots \longrightarrow \operatorname{Ext}_{K[Q]}^{*}\left(K^{(Q)}, K^{(Q)}\right) \longrightarrow \operatorname{Ext}_{K\left[Q_{1}\right]}^{*}\left(K^{\left(Q_{1}\right)}, K^{\left(Q_{1}\right)}\right) \\
& \operatorname{Ext}_{K\left[Q_{2}\right]}^{*}\left(K^{\left(Q_{2}\right)}, K^{\left(Q_{2}\right)}\right) \\
& \longrightarrow \operatorname{Ext}_{K\left[Q_{1} \cap Q_{2}\right]}^{*}\left(K^{\left(Q_{1} \cap Q_{2}\right)}, K^{\left(Q_{1} \cap Q_{2}\right)}\right) \longrightarrow \cdots
\end{aligned}
$$

qui est celle 5.16, d'après le Lemme 5 .

Pour établir notre lien, nous utilisons l'idée suivante: à tout recouvrement d'un poset par des sous-posets terminaux ("ouverts") est attaché un poset et vice-versa. Exactement: pour tout poset $Q$ et tout recouvrement terminal $P=\left\{Q_{1}, \ldots, Q_{n}\right\}$ de $Q$, il y a une relation canonique $\mathscr{R}: P \longrightarrow Q$, $\mathscr{R}\left(Q_{i}\right)=Q_{i}$ (P est le poset discret: $Q_{i} \geq Q_{j} \Longleftrightarrow Q_{i}=Q_{j}$ ). le poset $C_{\mathscr{R}}$ est alors appelé "graphe de $P$ ". Inversement: tout poset $R$ est le graphe 
d'un unique recouvrement terminal, de relation $\mathscr{R}: P \longrightarrow Q$ à savoir: $P$ est l'ensemble des éléments minimaux de $R$ (poset discret), $Q=R \backslash P$ et $\mathscr{R}(x)=\{y \in Q \mid y \geq x\}$.

5.17. ThÉORÈme 5 (Mayer-Vietoris). Si R est le graphe d'un recouvrement terminal $\left\{Q_{1}, \ldots, Q_{n}\right\}$ d'un poset $Q$, nous avons une suite exacte longue:

$$
\begin{aligned}
\cdots \longrightarrow \tilde{H}^{*}(K[R]) & \longrightarrow \tilde{H}^{*}(K[Q]) \\
& \longrightarrow \prod_{i=1}^{n} \tilde{H}^{*}\left(K\left[Q_{i}\right]\right) \longrightarrow \tilde{H}^{*+1}(K[R]) \longrightarrow \cdots
\end{aligned}
$$

où, pour toute $K$-algèbre $A, \tilde{H}^{0}(A)=\operatorname{coker}\left(K \longrightarrow H H^{0}(A)\right)$ et $\tilde{H}^{i}(A)=$ $H H^{i}(A)$ si $i \geq 1$.

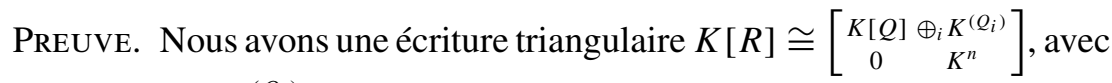
un bimodule $\bigoplus_{i} K^{\left(Q_{i}\right)}$ qui est $\left(K^{n}\right)^{o}$-plat. Nous appliquons le Théorème 1 aux $K[R]$-modules $\Lambda=\Lambda^{\prime}=K^{(R)}$ et obtenons une suite exacte longue $(* \geq 1)$ :

$$
\begin{aligned}
& \cdots \longrightarrow \operatorname{Ext}_{K[R]}^{*}\left(K^{(R)}, K^{(R)}\right) \\
& \longrightarrow \operatorname{Ext}_{K[Q]}^{*}\left(K^{(Q)}, K^{(Q)}\right) \longrightarrow \operatorname{Ext}_{K[Q]}^{*}\left(\bigoplus_{i} K^{\left(Q_{i}\right)}, K^{(Q)}\right) \longrightarrow \cdots
\end{aligned}
$$

Le Lemme 1 fournit un isomorphisme $\operatorname{Ext}_{K[Q]}^{*}\left(\bigoplus_{i} K^{\left(Q_{i}\right)}, K^{(Q)}\right) \cong$ $\prod_{i} \operatorname{Ext}_{K\left[Q_{i}\right]}^{*}\left(K^{\left(Q_{i}\right)}, K^{\left(Q_{i}\right)}\right)$ et le Lemme 5 nous ramène à 5.18: les termes en degré 0 nécessitent un léger aménagement (de là l'introduction de la cohomologie "réduite").

Notons que pour $i>g:=\operatorname{gldim}(K[R])$, nous avons $\tilde{H}^{i}(K[R])=0$ et, si $K$ est un corps, le morphisme $\tilde{H}^{g}(K[R]) \longrightarrow \tilde{H}^{g}(K[Q])$ (extrait de 5.19) est une surjection d'après la formule de Chase 3.7 (dans tous les cas $\operatorname{Ext}_{K\left[Q_{i}\right]}^{g}\left(K^{\left(Q_{i}\right)}, K^{\left(Q_{i}\right)}\right)=0$ ). C'est le cas particulier suivant qui permet d'interpréter 5.18 et, plus généralement, la suite exacte longue de Palmér-Roos (et donc celle de Happel) comme une suite de Mayer-Vietoris.

5.21. Corollarie 3. Pour $n=2$, la suite exacte longue 5.18 est celle de Mayer-Vietoris 5.16.

Preuve. Soit un recouvrement $Q=Q_{1} \cup Q_{2}$ par deux sous-posets terminaux et soit $R$ le graphe de ce recouvrement. Le corollaire est trivial si $Q_{1} \cap Q_{2}=\phi: R$ possède alors deux composantes connexes avec, chacun, un seul élément minimal. Dans les autres cas, nous avons une écriture triangulaire 
$K[R] \cong\left[\begin{array}{ccc}K[Q] & K^{\left(Q_{1}\right)} & K^{\left(Q_{2}\right)} \\ 0 & K & 0 \\ 0 & 0 & K\end{array}\right]$ ainsi qu'une suite exacte courte de $K[R]$-modules:

$$
0 \longrightarrow\left[\begin{array}{c}
K^{\left(Q_{1} \cap Q_{2}\right)} \\
0 \\
0
\end{array}\right] \longrightarrow\left[\begin{array}{c}
K^{\left(Q_{1}\right)} \\
K \\
0
\end{array}\right] \oplus\left[\begin{array}{c}
K^{\left(Q_{2}\right)} \\
0 \\
K
\end{array}\right] \longrightarrow\left[\begin{array}{c}
K^{(Q)} \\
K \\
K
\end{array}\right] \longrightarrow 0
$$

où ceux du milieu sont projectifs. Nous en déduisons des isomorphismes pour $* \geq 2$ :

$$
\begin{aligned}
\operatorname{Ext}_{K[R]}^{*}\left(K^{(R)}, K^{(R)}\right) & \cong \operatorname{Ext}_{K[R]}^{*-1}\left(K^{\left(Q_{1} \cap Q_{2}\right)}, K^{(R)}\right) \\
& \cong \operatorname{Ext}_{K\left[Q_{1} \cap Q_{2}\right]}^{*-1}\left(K^{\left(Q_{1} \cap Q_{2}\right)}, K^{\left(Q_{1} \cap Q_{2}\right)}\right) \\
& \cong H H^{*-1}\left(K\left[Q_{1} \cap Q_{2}\right]\right)
\end{aligned}
$$

provenant des Lemmes 1 et 5 . Pour les termes de bas degrés, nous obtenons une suite exacte

$$
\begin{aligned}
\left.0 \longrightarrow H H^{0}(K[R]) \longrightarrow \begin{array}{cc}
K\left[Q_{1}\right] & K^{\left(Q_{1}\right)} \\
0 & K
\end{array}\right] \\
H H^{0}\left[\begin{array}{cc}
K\left[Q_{2}\right] & K^{\left(Q_{2}\right)} \\
0 & K
\end{array}\right] \\
\longrightarrow H H^{0}(K[Q]) \longrightarrow H H^{1}(K[R]) \longrightarrow 0 .
\end{aligned}
$$

Ce qui prouve que nous avons un isomorphisme $H H^{*}(K[R]) \cong \tilde{H}^{*-1}\left(K\left[Q_{1} \cap\right.\right.$ $\left.Q_{2}\right]$ ). Il devient alors clair que la suite exacte 5.18 n'est rien d'autre que celle 5.16 .

\section{REFERENCES}

1. Auslander, M.,Comparison of global and algebra dimension, Nagoya Math. J. 11 (1957), 61-66.

2. Bendiffalah, B., Modules d'Extensions des Algèbres triangulaires, C. R. Acad. Sci. Paris, Ser. I 339 (2004), 387-390.

3. Bendiffalah, B., Guin, D., Cohomologie de l'algèbre triangulaire et applications, J. Algebra 282 (2004), 513-537.

4. Bendiffalah, B., Guin, D., Cohomologie des morphismes, Comm. Algebra 26-12 (1998), 3939-3951.

5. Bouc, S., Homologie de certains ensembles ordonnés, C. R. Acad. Sci. Paris, Ser. I-2 299 (2004), 49-52.

6. Cartan, H., Eilenberg, S., Homological Algebra, Princeton University Press, 1956.

7. Chase, S., A generalization of the ring of triangular matrix, Nagoya Math. J. 18 (1961), $13-25$.

8. Cibils, C., Tensor Hochschild homology and cohomology, Lecture Notes in Pure and Appl. Math. 210 (2000).

9. Dold, A., Lectures on Algebraic Topology, Springer-Verlag Berlin, 2nd edition, 1995. 
10. Fields, K. L., On the global dimension of residue rings, Pacific J. Math. 32-2 (1970), 345-349.

11. Fossum, R. M., Griffith, P. A., Reiten, I., Trivial extensions of abelian categories, Lecture Notes in Math. 456 (1975), 1-122.

12. Gerstenhaber, M., Shack, S. D., On the cohomology of an algebra morphism, J. Algebra 95-1 (1985), 245-262.

13. Gerstenhaber, M., Shack, S. D., On the deformation of algebra morphisms and diagrams, Trans. Amer. Math. Soc. 279-1 (1983), 1-50.

14. Gerstenhaber, M., Shack, S. D., Simplicial cohomology is Hochschild cohomology, J. Pure Appl. Algebra 30 (1983), 143-156.

15. Green, E. L., Solberg, $\varnothing$, Hochschild cohomology rings and triangular rings, Proceedings of the ninth international conference, Beijing 2000, p. 192-200. Beijing normal university press vol. II, 2002.

16. Happel, D., Hochschild cohomology of finite dimensional algebras, Lecture Notes in Math. 1404 (1989), 108-126.

17. Keller, B., Hochschild cohomology and derived Picard groups, J. Pure Appl. Algebra 190, 1-3 (2004), 177-196.

18. Loday, J-L., Cyclic Homology, 2nd edition, Grundlehren Math. Wiss. 301 (1998).

19. Löfwall, C., The global homological dimensions of trivial extensions of rings, J. Algebra 39 (1976), 287-307.

20. Michelena, S., Platzeck, M. I., Hochschild cohomology of triangular matrix algebras, J. Algebra, 233-2 (2000), 502-525.

21. Mitchell, B., Rings with Several Objects, Adv. Math. 8 (1972), 1-161.

22. Palmér, I., Roos, J-E., Explicit formula for the global homological dimensions of trivial extensions of rings, J. Algebra 27 (1973), 380-413.

23. Palmér, I., Roos, J-E.,Formules explicites pour la dimension homologique des anneaux de matrices généralisées, C. R. Acad. Sci. Paris A-273 (1971), 1026-1029.

24. Quillen, D., Homotopy properties of the poset of non-trivial p-subgroups, Adv. Math. 28 (1978), 101-128.

25. Reiten, I., Trivial extensions and Gorenstein rings, Thesis, University of Illinois, Urbana, 1971.

\author{
INSTITUT DE MATHÉMATIQUES ET DE MODÉLISATION DE MONTPELLIER (UMR CNRS 5149), \\ DÉPARTEMENT DE MATHÉMATIQUES, CASE 051 \\ UNIVERSITÉ MONTPELLIER II, \\ PLACE EUGÈNE BATAILLON, \\ 34095 MONTPELLIER CEDEX 5 \\ FRANCE \\ E-mail: ben@math.univ-montp2.fr
}

